of these homes are staffed by untrained nurses under the supervision of a trained nurse. The latter may have responsibility for several such homes. No longer are staff and patients visited routinely by the doctor. Instead, meetings are more likely to occur at times of crisis and thus, often result in the prescription of psychotropic drugs.

The prevalence of psychotropic drug use within adults with a learning disability living in the community, ranges between 14-36\% (Aman, 1987). Drugs are often used to treat behavioural disturbance rather than overt psychiatric illness, and ideally should only be prescribed after a full assessment and after, or in conjunction with, behavioural or social interventions.

Non-medical staff often appear antagonistic towards the use of psychotropic drugs, expressing concern that their patients are being 'doped' and subjected to unnecessary and distressing side effects. They are often unclear why particular drugs are being prescribed and are keen to know more. While most staff have access to a British National Formulary, the information is full of medical jargon and may be difficult to understand.

In an attempt to fill this gap in the literature and to reduce the misunderstandings within the staff team when psychotropic drugs are prescribed, I have produced a small drug information booklet for non-medical staff. It summarises the main considerations before prescribing and the indications and common side effects of antidepressants, lithium, antiepileptics, antipsychotics, benzodiazepines and beta-blockers. The booklets have been distributed to staff during small group teaching sessions on the subject. Initial feedback, from untrained nursing staff and other health care professionals such as psychologists, occupational therapists and social workers, has been extremely positive.

Jorsh (1991) suggests that psychiatrists should be more closely involved in the teaching of nursing students and states that it is important that people within a multidisciplinary team are able to use and understand a common language. The psychiatric registrar is in an ideal position to take on this valuable teaching role within the learning disability field and may, in addition, benefit immensely from the experience.

AMAN, M.G. (1987) Overview of pharmacotherapy: current status and future directions. Journal of Mental Defictency Research, 31, 121-130.

JORSH, M.S. (1991) The changes in the education of psychiatric nurses. Do psychiatrists have a role? Psychiatric Bulletin, 16, 339-340.

Gill SALMON, Registrar in Psychiatry, Oxfordshire Learning Disability Trust

\section{Adolescent psychiatric services}

Sir: Amidst the flurry of closures and growing concern about the future of dedicated adolescent psychiatric services, I am pleased to report that after many years of abortive attempts, Bristol will soon have its own adolescent in-patient and day patient service for the first time.

For more than 12 years, psychiatrists in the city have been working hard to develop such a unit. In spite of the well established local need and the hopeless situation of young people having to travel 60 miles or more for in-patient treatment, little progress has been made over the last decade. A major stumbling block has been the extreme difficulty in getting three independent health authorities to act in concert and agree on the funding, time-tabling and commissioning of any new service.

The advent of the NHS reforms and the purchaser/provider split allowed professionals in Bristol to channel their efforts towards the purchaser, and a helpful and co-operative series of planning meetings over the last 18 months has allowed the development to proceed for the first time. This joint planning between consultants from each of the three Bristol trusts and the purchaser, has enabled us to develop a sensible pragmatic operational policy with wholesale clinical support from potential referrers across the city. The purchaser was then able to invite local providers to put forward business cases for the unit and this process has now been completed.

The new unit will be developed by Frenchay Healthcare NHS Trust and, it is hoped, will open in April 1995. An ideal modern building has been found and within the next nine months a complete new adolescent psychiatric team will be recruited. I feel confident that this will lead to a significant improvement in services to this client group.

I strongly believe that the key to this success has been the close relationship with the purchaser. I would urge colleagues to be pro-active and energetic in developing this relationship. It is our experience that the development of a partnership with the purchaser, aimed at improving services for a neglected group, has led to this very positive outcome.

IAN Skeldon, Frenchay Healthcare Trust, Brtstol BS16 5EB

\section{Management of physical ill health in a psychiatric setting}

Sir: Psychogeriatric in-patients with functional and organic disorders on acute wards suffer from more physical disease than other people. There is a relationship between physical disorder and 
affective disorder in old age, probably with causality in both directions (Phillpott, 1990). Clearly physical ill health is an important factor in the elderly suffering with dementia.

The very high degree of physical morbidity was confirmed in a survey carried out by myself on in-patients in an acute functional and an acute dementia unit for the elderly which included 19 patients on the dementia ward and 22 patients on the functional ward. On the dementia ward each patient was on a mean of 4.8 medications of which 3.4 medications were prescribed for physical disorder. On the functional ward each patient was on a mean of $\mathbf{5 . 2}$ medications of which 2.9 were prescribed for physical disorder. On the dementia ward ten out of the 19 patients had an acute physical illness (as defined by onset within two weeks). Also in the dementia group a total of 59 chronic illnesses were detected (on average 3.1 chronic conditions per patient). There were no acute physical illnesses detected on the functional ward but there were 42 chronic physical conditions (an average of 1.9 chronic conditions per patient).

In view of these findings it is interesting to note that old age psychiatry wards are often staffed by registered mental nurses and psychiatric trainees. Generally a medical opinion may only be obtained by specific referral rather than regular liaison attendance or joint psychogeriatric- geriatric assessments. Junior medical staff, often in isolated psychiatric institutions, do have difficulty obtaining urgent medical opinions and may also meet a degree of prejudice. The management of physical ill health in the psychiatric setting is one that requires further investigation.

PHulport, R. (1990) Affective disorder and physical illness. International Clinical Psychopharmacology, B, Supplement 3.

S.F. Foster, Registrar in Psychiatry, Mersey Regional Rotation

\section{A study of the use of routine laboratory investigations in adult psychiatric patients}

Sir: The inappropriate use of laboratory tests observed in clinical practice (Sandler, 1979) appears to be a problem in psychiatric practice as well (White \& Barraclough, 1989).

We undertook a questionnaire study of adult psychiatric patients admitted to St Lukes Hospital, Middlesbrough during a three month period in 1992 to examine the use of laboratory tests and establish the pattern of physical testing in psychiatric practice. Of the 119 patients studied during this period, 43 were transferred or discharged without any diagnostic tests performed. Thirty-eight different tests were carried out on the remaining patients with a range of 1-12 tests per patient. Full blood count (66 tests) and biochemistry (SMAC) (74) were the most frequently requested tests. Thyroid functions tests (37), urine examination (13) and serum lithium levels (22) were also performed. Other physical tests were electroencephalograms (19), electrocardiograms (11), X-rays (8), serum drug levels in selfpoisoning (7).

The positive rate for full blood count was $18 \%$, biochemistry (SMAC) 24\%, thyroid function tests $13 \%$, urine $8 \%$ and serum lithium $32 \%$. More tests ( 3 tests/patient) were performed on patients who were older (46-65 years) and were in-patients (5 tests/patient) for a longer period ( 29 days or more). No significant differences were detected between males and females or between diagnostic groups.

The helpfulness of the tests was assessed by examining the clinical details in the patients' records; $80 \%$ of blochemistry tests were helpful, $57 \%$ of full blood counts, $100 \%$ of thyroid function tests and $79 \%$ of urine examinations. The reasons given for assessing the helpfulness of the tests were: exclusion of physical causes (23), estimation of therapeutic level (17), assessment of liver function (8) and adjustment of dosage of drugs (4).

This study demonstrates that laboratory tests are performed very frequently on psychiatric patients as a matter of practice and the pattern appears to be similar to that in other psychiatric hospitals and general medical wards. Training programmes for medical staff, protocols and guidelines, reviews and audit may help to minimise the inappropriateness of these tests.

SANDLER, G. (1979) Costs of unnecessary tests. British Medical Journal, u, 21-24.

WhITE, A.J. \& BARRACLOUGH, B. (1989) Benefits and problems of routine laboratory investigations in adult psychiatric admissions. Brittsh Journal of Psychiatry. 185, 65-72.

K.P. RAO, St Luke's Hospttal, Middlesbrough, Cleveland and R.S. RAMAIAH, Public Health Medicine, Walsall Health Authority, Walsall, West Midlands

\section{Psychiatrists and euthanasia}

Sir: Dr Huyse and colleagues have provided us with an interesting paper on 'Consultant-liaison psychiatrists and euthanasia in The Netherlands' (Psychiatric Bulletin, August 1994, 18, 497-500). However, euthanasia is not practised in most countries represented by the wide readership of the Bulletin. Indeed in the UK readers may be interested to know that the government has just accepted the recommendations of the House of Lords Medical Ethics Committee not to legalise euthanasia.

M. DOMINIC BEER, UMDS Guy's and Bexley Hospital, Bexley, Kent DA5 2BW 